\title{
Anti-leukemic activity of axitinib against cells harboring the BCR-ABL T315I point mutation
}

\author{
Seiichi Okabe*, Tetsuzo Tauchi, Yuko Tanaka, Juri Sakuta and Kazuma Ohyashiki
}

\begin{abstract}
The BCR-ABL; breakpoint cluster region-Abelson point mutation T315I is resistant to ABL tyrosine kinase inhibitors. However, axitinib, a vascular endothelial growth factor receptor inhibitor, is effective against this mutation. In this study, we investigated axitinib activity against ponatinib-resistant cells and found that axitinib inhibited cellular growth and apoptosis in Ba/F3 T315I-mutant cells and T315I-mutant primary samples, but not in ponatinib-resistant Ba/F3 cells and primary samples. Thus, an alternative strategy may be required to improve the prognosis of Philadelphia-chromosomepositive leukemia patients harboring BCR-ABL point mutations.
\end{abstract}

Keywords: Axitinib, Resistant cell, Ponatinib, T315I, Compound mutation

\section{Letters to the editor}

The BCR-ABL1 fusion gene is a causative oncogene in chronic myeloid leukemia (CML) and $30-50 \%$ of acute lymphoblastic leukemia cases $[1,2]$. Although ABL tyrosine kinase inhibitors (ABL TKIs) such as imatinib, nilotinib, dasatinib, and bosutinib have improved CML treatment [3], such therapies cannot cure patients with Philadelphia chromosome (Ph)-positive leukemia because of leukemia stem cells [4]. Moreover, some patients develop $\mathrm{BCR}-\mathrm{ABL}$ point mutations and become resistant to $\mathrm{ABL}$ TKI therapy [5]. In particular, the ABL kinase domain mutation T315I is resistant to imatinib and second-generation ABL TKIs (e.g., nilotinib, dasatinib, and bosutinib). Accordingly, this mutation is often found in patients with TKIresistant disease [6]. A third-generation ABL TKI, ponatinib, and omacetaxine which is a semisynthetic form of homoharringtonine, was recently developed [7]. Ponatinib is a potent oral tyrosine kinase inhibitor that affects both unmutated and mutated BCR-ABL [8]; it is effective against T315I-mutant cells and has been approved for TKI-resistant or intolerant CML and Ph-positive ALL patients. Omacetaxine is approved for the treatment of chronic or accelerated-phase CML refractory to TKIs [7].

Recently, the vascular endothelial growth factor receptor (VEGFR) inhibitor axitinib was found to exhibit antileukemic activity against T315I-mutant disease. In the

\footnotetext{
* Correspondence: okabe@tokyo-med.ac.jp

Department of Hematology, Tokyo Medical University, 6-7-1 Nishi-shinjuku, Shinjuku-ku, Tokyo 160-0023, Japan
}

comparative effectiveness of axitinib versus sorafenib in advanced renal cell carcinoma (AXIS) trial [9], axitinib improved progression-free survival (PFS) compared to sorafenib, which is an all-multikinase inhibitor that blocks angiogenesis targets [10], in patients with advanced renal cell carcinoma (RCC). Axitinib was approved for the treatment of advanced RCC. Axitinib is an orally active and potent TKI of VEGFRs 1,2 , and 3 and inhibits BCR-ABL1, especially the T315I variant, via a distinct binding conformation [11]. In this study, we investigated whether axitinib could suppress ponatinibresistant compound-mutant cells harboring the T315I mutation. A 72-h axitinib treatment inhibited the growth of $\mathrm{Ba} / \mathrm{F} 3 \mathrm{~T} 315 \mathrm{I}$ cells (Fig. 1a). Immunoblot analysis of axitinib-treated cells revealed dose-dependent decreases in BCR-ABL, the downstream molecule Crk-L, and ribosomal S6 protein phosphorylation and increases in caspase 3 and Poly (ADP-ribose) polymerase (PARP) activity (Fig. 1b, c, e). Ponatinib and axitinib also induced apoptosis, significantly increased caspase activity (Fig. 1d), and reduced Akt activity (Fig. 1f).

In contrast, clinically available concentrations of axitinib did not inhibit the growth of ponatinib-resistant $\mathrm{Ba} / \mathrm{F} 3$ cells (Fig. 1a). Immunoblot analysis revealed that BCR-ABL, Crk-L, and S6 kinase phosphorylation were not inhibited by axitinib or ponatinib (Fig. 1b, e). Similarly, no increase in caspase activity or decrease in Akt activity was observed following axitinib treatment (Fig. 1c, 


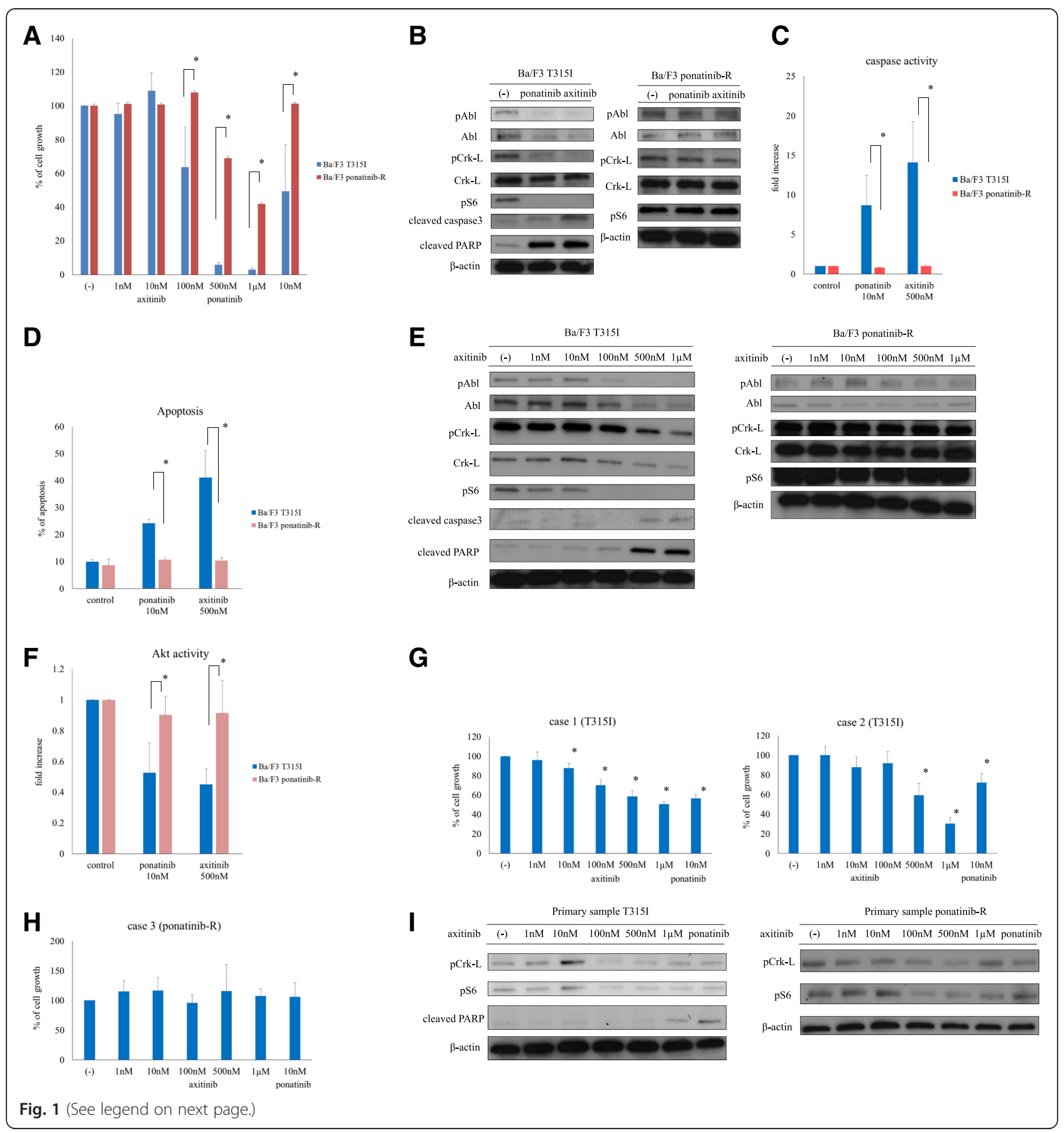


(See figure on previous page.)

Fig. 1 Growth inhibition and cellular signaling following axitinib/ponatinib treatment in T315I-mutant and compound-mutant cells. a Ba/F3 T315I or Ba/F3 ponatinib-resistant (Ba/F3 ponatinib-R) cells were exposed to axitinib or ponatinib for $72 \mathrm{~h}$ at the indicated concentrations and subjected to quantitative cell proliferation analysis. Each result is presented as the mean percentage of proliferation relative to unexposed control cultures. ${ }^{*} P<0.05$ compared to T315l cells. b Ba/F3 T315l or Ba/F3 ponatinib-R cells were treated with ponatinib (10 nM) or axitinib (500 nM) for $24 \mathrm{~h}$. Total cell extracts were examined via immunoblot analysis with anti-phospho ABL, phospho-Crk-L, phospho-S6, cleaved caspase 3, cleaved PARP, ABL, Crk-L, and $\beta$-actin antibodies. c Caspase activity was analyzed using an ApoAlert ${ }^{\circledR}$ Caspase-3 Colorimetric Assay Kit (Takara Bio Inc. Otsu, Shiga, Japan) according to the manufacturer's protocol. Data represent three independent sets of experiments. ${ }^{*} P<0.05$ compared to Ba/F3 T315l and $\mathrm{Ba} / \mathrm{F3}$ ponatinib-R cells. d Apoptosis in Ph-positive cell lines was assayed using a FITC Annexin V Apoptosis Detection Kit Im (BD Pharmingen, San Jose, CA, USA). The experiments were performed in triplicate. e Ba/F3 T315I or Ba/F3 ponatinib-R cells were treated with axitinib at the indicated concentrations for $24 \mathrm{~h}$. Total cell extracts were examined via immunoblot analysis with anti-phospho ABL, phospho-Crk-L, phospho-S6, cleaved

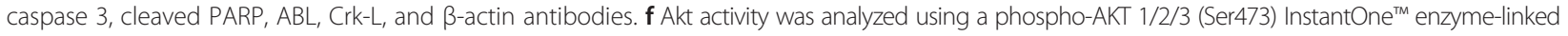
immunosorbent assay kit (Affymetrix, Cleveland, OH, USA) according to the manufacturer's protocol. Data represent three independent sets of experiments. ${ }^{*} P<0.05$ compared to Ba/F3 T315I and Ba/F3 ponatinib-R cells. $\mathbf{g}, \mathbf{h}$ T315I-positive or compound-mutant primary cells were subjected to quantitative cell proliferation analysis after a 72-h exposure to axitinib or ponatinib. Each result is presented as the mean percentage of proliferation relative to unexposed control cultures. ${ }^{*} P<0.05$ compared to control. i T315I-positive or compound-mutant primary cells were treated with axitinib at the indicated concentrations for $24 \mathrm{~h}$. Total cell extracts were examined via immunoblot analysis with anti-phospho-Crk-L, phospho-S6, cleaved PARP, and $\beta$-actin antibodies

f), and neither ponatinib nor axitinib affected apoptosis in these cells (Fig. 1d).

We next evaluated primary T315I-mutant and ponatinibresistant compound-mutant samples. Axitinib potently inhibited the growth of T315I-mutant primary cells in a dose-dependent manner (Fig. 1g). Immunoblot analysis further revealed reduced Crk-L and S6 kinase phosphorylation after axitinib or ponatinib treatment (Fig. 1i). In contrast, the growth of ponatinib-resistant primary cells was not affected by ponatinib or axitinib (Fig. 1h). Immunoblotting revealed that neither ponatinib nor axitinib affected the phosphorylation of Crk-L and S6 kinase in ponatinibresistant cells (Fig. 1i).

In CML, ABL TKI resistance is frequently caused by ABL kinase domain mutations. The T315I mutation is resistant to all ABL TKIs except ponatinib. However, we previously described ponatinib-resistant cells resulting from a BCR-ABL compound mutation [12]. Although axitinib, which is currently being investigated for efficacy in patients with Ph-positive T315I-mutant leukemia, induced apoptosis in T315I-mutant cells, it was ineffective against cells with a compound mutation including T315I. An alternative strategy will be required to improve the prognosis of patients with Ph-positive, BCR-ABL-mutant leukemia. Current evidence to direct the management of ABL TKI-resistant disease, particularly those harboring T315I and compound mutations, is limited. New molecular-targeted drugs and an understanding of ABL TKI resistance mechanisms are required to apply an appropriate therapeutic approach.

\section{Abbreviations}

CML: chronic myeloid leukemia; Ph: Philadelphia chromosome; TKI: tyrosine kinase inhibitor; VEGFR: vascular endothelial growth factor receptor.

\section{Competing interests}

Kazuma Ohyashiki received research support from Novartis and BMS.

\section{Authors' contributions}

$\mathrm{SO}$ performed the experimental procedures; $\mathrm{T}, \mathrm{YT}, \mathrm{JS}$, and $\mathrm{KO}$ designed and coordinated the study and interpreted data. All authors have read and approved the final manuscript.

\section{Acknowledgements}

This work was supported by a High-Tech Research Center Project for private universities, a matching fund subsidy from the Ministry of Education, Culture, Sports, Science, and Technology (MEXT), and the University-Industry Joint Research Project for private universities, a matching fund subsidy from MEXT. This work was also supported by Grants-in-Aid for Scientific Research from MEXT and Supporting Positive Activities for Female Researchers. We also thank the Tokyo Medical University Research Center for technical support.

Received: 7 July 2015 Accepted: 17 July 2015

Published online: 04 August 2015

\section{References}

1. Rowley JD. Letter: a new consistent chromosomal abnormality in chronic myelogenous leukemia identified by quinacrine fluorescence and Giemsa staining. Nature. 1973;243:290-3.

2. Kurzrock R, Shtalrid M, Talpaz M, Kloetzer WS, Gutterman JU. Expression of c-abl in Philadelphia-positive acute myelogenous leukemia. Blood. 1987;70:1584-8.

3. Sweet $K$, Zhang L, Pinilla-lbarz J. Biomarkers for determining the prognosis in chronic myelogenous leukemia. J Hematol Oncol. 2013;6:54.

4. Ahmed W, Van Etten RA. Alternative approaches to eradicating the malignant clone in chronic myeloid leukemia: tyrosine-kinase inhibitor combinations and beyond. Hematology Am Soc Hematol Educ Program. 2013;2013:189-200.

5. Kantarjian HM, Talpaz M, Giles F, O'Brien S, Cortes J. New insights into the pathophysiology of chronic myeloid leukemia and imatinib resistance. Ann Intern Med. 2006;145:913-23.

6. Ravandi F. Managing Philadelphia chromosome-positive acute lymphoblastic leukemia: role of tyrosine kinase inhibitors. Clin Lymphoma Myeloma Leuk. 2011;11:198-203.

7. Lü S, Wang J. Homoharringtonine and omacetaxine for myeloid hematological malignancies. J Hematol Oncol. 2014;7:2.

8. Cortes JE, Kantarjian H, Shah NP, Bixby D, Mauro MJ, Flinn I, et al. Ponatinib in refractory Philadelphia chromosome-positive leukemias. N Engl J Med. 2012:367:2075-88.

9. Rini Bl, Escudier B, Tomczak P, Kaprin A, Szczylik C, Hutson TE, et al. Comparative effectiveness of axitinib versus sorafenib in advanced renal cell carcinoma (AXIS): a randomised phase 3 trial. Lancet. 2011;378:1931-9.

10. Smith AD, Roda D, Yap TA. Strategies for modern biomarker and drug development in oncology. J Hematol Oncol. 2014;7:70. 
11. Pemovska T, Johnson E, Kontro M, Repasky GA, Chen J, Wells P, et al. Axitinib effectively inhibits BCR-ABL1(T315I) with a distinct binding conformation. Nature. 2015;519:102-5.

12. Okabe S, Tauchi T, Tanaka Y, Katagiri S, Kitahara T, Ohyashiki K. Activity of omacetaxine mepesuccinate against ponatinib-resistant BCR-ABL-positive cells. Blood. 2013;122:3086-8.

Submit your next manuscript to BioMed Central and take full advantage of:

- Convenient online submission

- Thorough peer review

- No space constraints or color figure charges

- Immediate publication on acceptance

- Inclusion in PubMed, CAS, Scopus and Google Scholar

- Research which is freely available for redistribution 